\title{
Teoría y praxis: \\ el deporte como objeto de estudio \\ y como objeto de enseñanza
}

\author{
Theory and praxis: sport as an object of study \\ and as a teaching subject
}

\section{José Luis Corbo*}

\footnotetext{
* Licenciado en Educación Física (ISEF-UdelaR). Magíster en Didáctica de la Educación Superior (Universidad CLAEH). Doctorando en Ciencias de la Actividad Física y el Deporte (Universidad Autónoma de Madrid). Director coordinador de Educación Física del Consejo de Educación Inicial y Primaria (CEIP-ANEP).

-joselocorbo@gmail.com http://orcid.org/0000-00026076-3451
}

\section{Resumen}

El presente ensayo problematiza el lugar de la producción de conocimiento sobre deporte y las posibles $-\mathrm{y}$ necesarias - relaciones entre deporte y la enseñanza de este como contenido de la educación física. En ese sentido, y problematizando los sentidos educativos de la enseñanza del deporte, se desarrolla la relación teoría-praxis en línea con la epistemología del materialismo histórico, avanzando desde Marx, pasando por la Escuela de Frankfurt, Gramsci y Althusser y abordando la problemática de la enseñanza del deporte de la mano de los autores contemporáneos más representativos en el ámbito local e internacional. A la vez, se proponen articulaciones entre académicos y profesionales con la intención de promover el potencial agencial en la construcción de las prácticas docentes de ambos campos.

Palabras clave: enseñanza, deporte, educación física.

\section{Abstract}

This essay problematizes the place of knowledge production both in the field of sport and about sport, as well as the possible - and necessary - relationships between production and teaching of sport as a content in Physical Education. Problematizing the educational senses of the teaching of sport, the theory-praxis relationship is developed in line with the epistemology of 
historical materialism, advancing from Marx, passing through the Frankfurt School, Gramsci and Althusser, and addressing the problem of the teaching of sport at the hand of the most representative contemporary authors in the local and international spheres. At the same time, articulations between academics and professionals are proposed with the intention of promoting their potential agency in the construction of teaching practices in both fields.

Keywords: teaching, sport, physical education.

\section{Introducción}

El deporte es, sin lugar a dudas, uno de los objetos culturales más trascendentes de fines del siglo XX y principios del XXI. Su significación ha sido tal que la magnitud de su alcance es poco menos que comparable con los fenómenos religiosos. Los deportistas adquieren rasgos míticos y la práctica deportiva trasciende cualquier tipo de fronteras, determinando entonces diversidad de construcciones y variedades de formas y sentidos a partir de los significados que cada sociedad le asigna.

A su vez, desde hace mucho tiempo, la industria cultural se ha apropiado del deporte (Adorno y Horkheimer, trad. 2016), transformándolo en objeto de consumo y acentuando sus rasgos explícitos del mercado deportivo a partir de lo implícito de la dialéctica estructura-superestructura (Marx, trad. 1989). En esa perspectiva, el deporte se mueve al ritmo de las relaciones productivas, las que definen formas y sentidos, y el objeto superestructural deporte aporta a su vez a las lógicas de aquellas relaciones de producción. El valor de cambio del deporte es por tanto fundamental en esta dinámica productiva.

Como respuesta a esa historia reciente, mucho se estudia y mucho se dice sobre deporte. La producción de conocimiento, incluso aquella ligada a la educación física, ha entendido que el saber del deporte es mucho más que el saber cómo jugarlo (Velázquez Buendía, 2004; Sarni y Corbo, 2021) y que su comprensión deberá entonces trascender los límites de su práctica, indagando en sus sentidos generales y particulares. Un segundo desafío dialéctico.

Es así como el campo del deporte se puebla de una diversidad de interpretaciones que se mueven desde unas que defienden su potencial axiológico como bandera del deporte moderno inglés, hasta otras tantas que lo demonizan. Estas últimas devienen de análisis estructuralistas que definen pares antinómicos, cosificando a los objetos en el sentido hegeliano, es decir, estableciendo definiciones rígidas y estáticas que desconocen el devenir dialéctico de las prácticas y por tanto de los conceptos (Jameson, 2013), 
desconociendo a su vez las posibilidades para la acción transformadora de los sujetos sobre los objetos.

El hecho es que cuando se trata de deporte y educación en los sistemas educativos formales, cuando se habla de la inclusión del deporte como contenido curricular, las relaciones entre la producción de conocimiento y la acción educativa son también de dependencia dialéctica. Lo que se produce en relación con el saber del deporte condiciona inevitablemente lo que sobre el deporte se enseña en la escuela, en el liceo, y donde se enseña a quienes enseñarán deporte, es decir, en la formación de profesores y licenciados en educación física.

En dicho sentido, es claro que los que demonizan el deporte proponen su exclusión de los espacios educativos formales, argumentando los problemas que esconden sus formas y sentidos, funcionales al modelo productivo capitalista y a otras tantas relaciones sociales injustas propias de los sistemas neoliberales. Desde esa perspectiva, no hay posibilidad de transformación desde la acción educativa, ya que, si aquello que es sometido a juicio crítico por la academia es excluido de los diseños curriculares, la transformación quedaría fuera de la educación porque los sujetos de educación no accederían al conocimiento y a la manipulación del objeto deporte y mucho menos serían agentes de cambio.

Desde otra perspectiva, el propio Marx expresaba en su Exposición ante el Consejo General de la Asociación Internacional de Trabajadores (AIT) en 1869:

El ciudadano Marx afirma que una dificultad de índole peculiar está ligada a esta cuestión. Por una parte, es necesario cambiar las condiciones sociales para crear un nuevo sistema de enseñanza; por otra, hace falta un sistema de enseñanza nuevo para poder cambiar las condiciones sociales (Marx y Engels, trad. 1978, p. 9)

El problema parece cobrar luz a partir de lo expresado. Está claro que la educación y los procesos de enseñanza implícitos en ella son el producto de una sociedad que define sentidos e intereses (Habermas, 1986). La lucha de clases subyace a las formas de la educación e imprime los sentidos de las prácticas educativas. Por otra parte, sabemos también que, para cambiar esa sociedad que produce una educación que a su vez reproduce desigualdad, es necesario cambiar la educación. Nos encontramos entonces con un nuevo problema de dependencia dialéctica.

Entendemos entonces, y a la luz de las anteriores reflexiones, que el papel de la academia en la producción de conocimiento es fundamental en el sentido que lo expresaba Habermas (1995), produciendo para transformar y en la búsqueda de la justicia social objetiva (práctica/concreta), y que las posibilidades de transformación solamente aparecen cuando se produce conocimiento con la intención explícita de habilitar las posibilidades de trasformación sobre la praxis. 


\section{Teoría y praxis}

\section{El legado marxista y neomarxista}

Entendemos la praxis como aquella acción de un sujeto sobre otro sujeto con la intención de transformar y de transformarse, y a partir de una necesaria distinción con la poiesis como práctica productiva, como la acción de uno o más sujetos sobre uno o más objetos con la intención de obtener un producto, tal como lo expresaba Althusser (2015) a partir de lo presentado por Aristóteles en el apartado 4 del libro sexto de su Ética a Nicómaco (trad. 2019).

La praxis transformadora opera, como decía Freire (1969), actuando de forma consciente, políticamente consciente, buscando incidir sobre las relaciones de los sujetos y en la búsqueda de la justicia social, la que deberá, como decíamos, ser material, objetiva. Las desigualdades de orden material se resuelven en el terreno de lo material; la libertad ideal, la que promueven las filosofías liberales y neoliberales, es por tanto una libertad de pensamiento que ni siquiera como pensamiento es libre, ya que es el ser social y las prácticas que en él se inscriben, los que determinan las formas de la conciencia (Marx, trad. 1989).

En esta línea, Adorno y Horkheimer -director y exdirector de la escuela de Frankurt- discutían en 1956 las relaciones de su producción académica con la praxis, ante la inquietud de un Adorno que se sentía productor de un sinnúmero de conocimientos, pero que a veces temía que le entregaran una institución del Estado para manejar y no poder reflejar su producción en la transformación de la praxis; a lo que Horkheimer respondía:

La teoría es teoría en sentido propiamente dicho sólo allí donde sirve a la praxis. La teoría que pretende ser suficiente en sí misma es mala teoría. Por otro lado, es también mala teoría cuando sólo está ahí para producir algo. (Adorno y Horkheimmer, trad. 2014, p. 56)

Es decir que, ante un inminente avance de las filosofías idealistas, esencialmente de una nueva ontología a partir de Heidegger - para quien el propio Adorno dedicaría un seminario en 1960 (2017) - , los integrantes de la escuela de Frankfurt buscaban recuperar la tesis $\mathrm{XI}^{1}$ de Marx a partir de una filosofía que pensara el mundo y sus prácticas desde una producción teórica en dependencia dialéctica con la praxis, una suerte de teoría que se enfrentara al mundo práctico con posibilidades de transformarlo.

1 Los filósofos no han hecho más que interpretar de diversos modos el mundo, pero de lo que se trata es de transformarlo» (Marx, 1969). 
Si bien este análisis refiere especialmente a lo que los idealismos representaron para la historia de la filosofía, no parece para nada anacrónico traerlo al universo actual de la producción académica donde, como decíamos, el conocimiento en torno del objeto que nos ocupa, el deporte, alcanza niveles cuantitativos inéditos pero alejados en el mayor de los casos de las posibilidades de retorno sobre la praxis, es decir, de la habilitación de la posibilidad dialéctica para la transformación de la práctica deportiva y de la cultura deportiva.

\section{Deporte y praxis}

Corresponde entonces, y en la misma línea de pensamiento, ocuparnos del deporte como contenido de enseñanza de la educación física en las instituciones educativas formales. Parece de más afirmar que, para que el deporte sea problematizado para ser enseñado, es apremiante recuperar el vínculo entre la producción de conocimiento y la enseñanza, lo que si bien suena de perogrullo, no resulta tan sencillo al momento de construir la relación.

El propio Adorno (trad. 1998) expresó en relación con los «tabúes sobre la profesión de enseñar», su preocupación por la distancia que se dibujaba entre el académico y el enseñante, el evidente desprestigio al que se somete el que enseña y el enorme podio en el que se encumbra al que produce. No obstante, como decíamos, el saber de deporte implica la articulación de saberes de diversos campos académicos, que construyen al objeto a partir de dependencia dialécticas y, por tanto, indisociables.

Particularmente, pensando en el deporte y al momento de enseñarlo, los debates al interior de las prácticas son analizados y problematizados al interior de la academia, construyendo nuevos discursos, algunos estrictamente interpretativos y otro tanto críticos y transformadores.

En el abanico de prácticas, los opuestos nos muestran aquellos docentes que, en el intento de alejar las formas y sentidos del deporte espectáculo de la escuela proponen enseñar otra cosa, es decir, darle la espalda a la realidad y construir una realidad paralela, un deporte que exista estrictamente para las instituciones educativas, alineados con los teóricos que proponen un deporte de la escuela (Gómez, 2002). Del otro lado, tenemos a aquellos que entendemos que se debería tomar el deporte que efectivamente existe en el mundo, con sus virtudes y pesares, problematizarlo, abordar sus contradicciones, es decir, enseñarlo críticamente en virtud del potencial agencial de los estudiantes. En definitiva, poner al deporte en la escuela (Sarni y Corbo, 2021).

Por lo tanto, una enseñanza del deporte que piense el deporte en concreto, es decir, que se ocupe de una práctica deportiva que en efecto existe más allá del patio escolar, deberá partir de una pregunta en extremo básica pero no por eso menos compleja: 
¿con qué deporte convivimos y qué deporte enseñamos? A partir de la primera: ¿debemos pensar en la enseñanza de un deporte de la escuela o en la enseñanza y problematización del deporte en la escuela?

Ambas preguntas están inexorablemente ligadas. Si no nos preguntamos con qué deporte convivimos, parece de orden afirmar que el primer paso es empezar a conocerlo, ya que es poco menos que evidente que resultará imposible problematizar en la escuela algo sobre lo que no sabemos, ya que reproducción antecede a transformación.

Si nos proponemos, por otra parte, pensar en un deporte que sea solamente de la escuela, estaríamos limitando nuestra enseñanza a la construcción de un objeto inexistente, es decir, trasformando la escuela en un espacio de supuesta abstracción, renunciando entonces así a cualquier posibilidad transformadora.

Por otra parte, si recuperamos la dialéctica entre lo abstracto y lo concreto, podríamos decir que aquello que a simple vista se presenta como abstracción, desconectado del mundo real, siempre estará dialécticamente vinculado a aquel, por lo cual, lo que no transforma reproduce. Es ilógico a su vez enseñar algo que no existe, si lo que pretendemos es transformar, ya que pensar lo inexistente no implica bajo ninguna perspectiva transformación alguna.

Esta discusión es tan vieja como la filosofía y no se encuentra tan alejada de aquello que planteaban Adorno y Horkheimer. Desde una perspectiva epistemológica, podemos decir que la encrucijada se define de la siguiente manera:

- Por un lado, la tesis idealista plantea partir de una idea, de lo no existente en el plano material, para eliminar lo existente por considerarlo malo (lo afirmativo o hegemónico en el plano filosófico o lo negativo en el universo de lo ético (Dusell, 2016). En ese sentido, propone un deporte diferente y mejor a partir de parámetros axiológicos establecidos a priori, propone construir un deporte de la escuela.

- Por otra parte, la tesis materialista — a la que adherimos-, y a partir de la teoría crítica, propone partir de la práctica, es decir, del deporte que la sociedad construye y que existe como fenómeno superestructural, problematizarlo a partir de su análisis, desentrañando sus sentidos desde la crítica inmanente, esto es, desde el análisis de sus propias contradicciones -el objeto que enfrenta al propio objeto-, para salir fuera de él a enfrentar el modelo productivo del que el deporte depende dialécticamente (Adorno, trad. 2015). Propone entonces abordar al deporte en la escuela para su crítica y transformación y pensar en las formas de una educación que trasciende al propio objeto, al objeto en sí, y que postule la necesidad de abordar críticamente el universo de las prácticas sociales. 
En esta perspectiva, podríamos decir que sería inocente para la propia educación física establecer límites epistémicos con relación al campo y ocuparse celosamente de la defensa de su espacio. Más aún en momentos en que cualquier intento por establecer esos límites en relación con la producción de conocimiento se presenta por lo menos difuso.

El saber del deporte, entonces, deberá nutrirse de los saberes de las ciencias sociales - filosofía, sociología, antropología y psicología-, de la pedagogía y la política inexorablemente ligadas-y de la didáctica, ocupada esencialmente en el cómo enseñar aquello que se pretende transformador, didáctica que, de desligarse del resto de las ciencias, caería inevitablemente en el terreno de la discusión del método y, por tanto, en la tan temida reproducción de formas y sentidos.

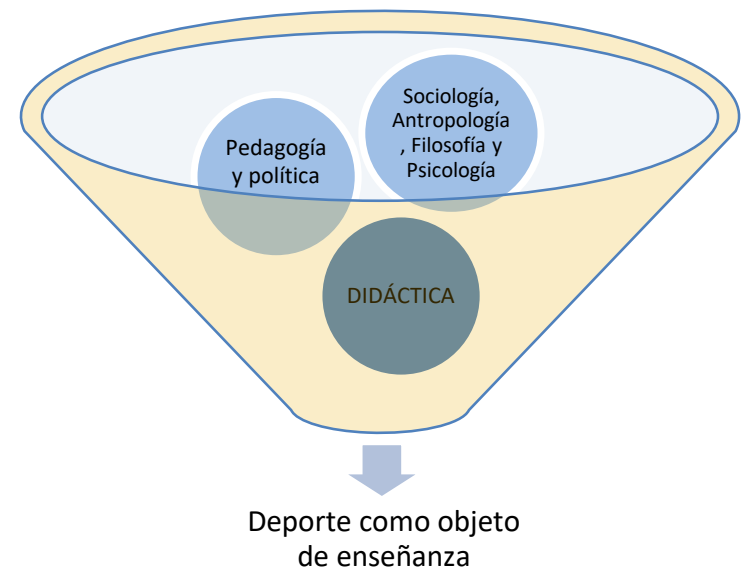

Figura 1. La construcción epistémica del objeto deporte (elaboración propia).

La imagen representa las necesidades que demanda la construcción del deporte como objeto de enseñanza, las que solamente serán atendidas en la medida en que el conocimiento no sea fragmentario, sino que se construya a partir de la articulación, complementaria y siempre dinámica, de aquello que las ciencias sociales aportan sobre el hombre y la sociedad, las relaciones políticas que subyacen a la trama social y a la pedagogía, y la didáctica específica del área. 


\section{Los estudios sobre deporte: relaciones dialécticas (la teoría y la praxis, lo abstracto y lo concreto)}

Como decíamos, el deporte es parte de una trama de relaciones de dependencia que determinan su ser a la vez que, dada su magnitud como fenómeno superestructural, lo transforman en un objeto capaz de determinar también las relaciones entre sujetos siendo así, inclusive, peligrosamente funcional a los modelos productivos.

Asignar al deporte un valor educativo propio, repetir asépticamente el discurso de los valores en el deporte sin problematizar su génesis, es decir, su nacimiento ligado a la escuela deportiva de Thomas Arnold y los sentidos a los que obedecía en su época, es por lo menos peligroso, dado el potencial reproductivo de sus prácticas y sus rasgos constitutivos, funcionales al viejo capitalismo europeo y, más aún, al capitalismo neoliberal del siglo XXI.

Por lo tanto, producir conocimiento sobre deporte implicará considerar como punto de partida el universo de las prácticas, atendiendo lo universal y lo particular, haciendo abstracción del objeto deporte para su análisis y problematización y construyendo el saber del objeto desde lo abstracto hacia lo concreto. La construcción epistemológica demanda inevitablemente la abstracción del objeto, pero la necesidad de recuperar la relación teoría-praxis demanda a su vez el retorno sobre lo concreto para la construcción de sus sentidos — pedagógicos-y de sus prácticas de enseñanza.

Es decir que, para el campo de la educación física, es impostergable el vínculo entre lo producido en la academia y lo enseñado en las instituciones, ya que es rasgo constitutivo del campo su dimensión pedagógica (Bracht, 2005); producir para no enseñar parecería en principio contradictorio.

Podemos afirmar entonces que es menester de la educación física, de la producción de conocimiento y de la enseñanza evitar la cosificación del deporte, es decir, la conceptualización rígida de un objeto que es dinámico, que se encuentra en permanente transformación (figura 2). Su problematización y crítica estará limitada por las posibilidades de contemplación y seguimiento de ese devenir dialéctico al que los objetos sociales están infinitamente sometidos.

Agregamos, además, que el deporte como concepto es una abstracción, una idea que sólo se significa en el universo de lo concreto en la medida en que se define como práctica, en la medida en que se le asigna temporalidad y espacialidad al objeto, ubicando la práctica en relación con la totalidad de las prácticas con las que se relaciona y que aportan a la construcción de sus formas y sentidos, es decir, ubicándolo en el mundo real. 


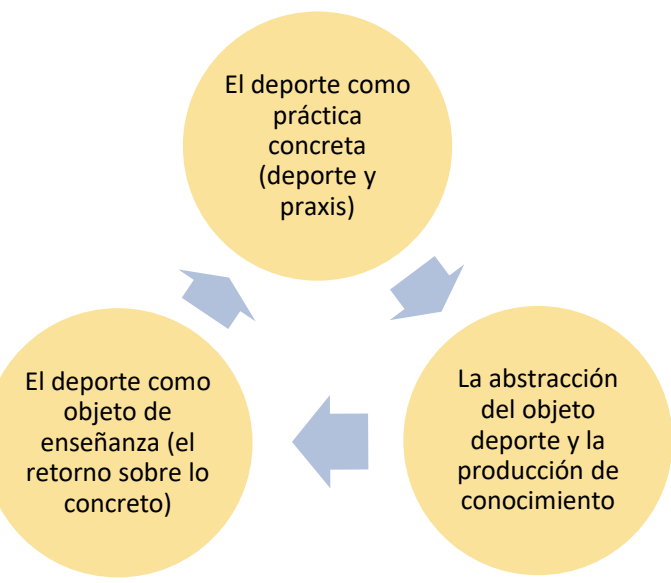

Figura 2. Relaciones teoría-praxis (elaboración propia)

\section{El deporte como contenidlo de enseñanza de la educación física: los estudios en deporte}

Entendemos, continuando con las relaciones entre producción de conocimiento y enseñanza, que pensar en el deporte en la educación formal implica:

- Pensarlo como fenómeno superestructural, en dependencia dialéctica con las relaciones de producción. Esto implica alejarse de las teorías idealistas que presentan a la cultura como fenómeno aislado, desconectado de la base material, y adherir a una concepción de cultura que se mueve en dependencia con un mercado que la produce y reproduce, cultura que es tanto más significativa en cuanto sus rasgos miméticos y catárticos son potenciados (Elias y Dunning, 1992).

- Comprender que su lugar como contenido de la educación física lo ha cargado de roles funcionales y secundarios, despojándolo de sus relaciones: se ha presentado siempre como un objeto presuntamente abstracto (se enseña pero no se dice para qué se enseña, así como tampoco se piensa para qué se enseña).

- Problematizarlo como fenómeno concreto, evitando tanto caer en idealismos a partir de la enseñanza de objetos no existentes como en la enseñanza de otros objetos, dada la manipulación de sus elementos constitutivos: atender su vigilancia epistemológica (Chevallard, 1998). 
- Superar la enseñanza de la dimensión del practicante deportivo, atendiendo a su vez a las demás dimensiones que determinan sus rasgos como fenómeno histórico (Velázquez Buendía, 2004).

Por lo tanto, entendemos que si la producción de conocimiento en educación física sobre el objeto deporte demanda de la atención prioritaria de sus sentidos educativos, es decir del por qué y para qué enseñarlo, con qué sentidos, será necesario prestar atención a aspectos medulares del proceso de producción, tomando como punto de partida y de forma impostergable el abordaje explícito de las relaciones de dependencia en muchos casos relegadas, y atendiendo siempre a la transformación permanente de las prácticas de los docentes con sus estudiantes.

Referimos a continuación a, por lo menos, algunas de estas relaciones:

1. Dependencia y complementariedad en la producción: Estudios sobre deporte (sociología, antropología, filosofía, psicología) y estudios en la educación y en la enseñanza del deporte (pedagogía y didáctica).

2. Posibilidades de agencialismo tanto de la academia como de los docentes y estudiantes en relación con el deporte como fenómeno social y cultural. Atención particular al lugar de los intelectuales en los procesos revolucionarios (Gramsci, trad. 2015).

3. Comprensión y crítica de relaciones estructurales a partir de la comprensión y crítica de los fenómenos superestructurales: pensar en una praxis transformadora. Todo abordaje crítico es insuficiente si no deviene en la crítica al modelo productivo, al capitalismo neoliberal y a sus formas de reproducción de la desigualdad.

A su vez, es preciso destacar que los rasgos del deporte que se enseña se vinculan necesariamente con el proyecto político para el cual se enseña, existiendo entonces criterios apriorísticos que se naturalizan —en el sentido habermasiano (1986) - en función del espacio en donde el deporte se configura y que, por tanto, deberán ser también problematizados.

En rasgos generales, destacamos a continuación algunas distinciones que entendemos ilustrativas para pensar la enseñanza del deporte específica y enfáticamente para cada caso concreto de los que se plantean:

- El proyecto político educativo define los intereses sobre la enseñanza del deporte (técnico, práctico y crítico) (Grundy, 1987).

- Los intereses técnicos (clubes deportivos) se limitan a la producción de deportistas (poiesis). 
- Los intereses prácticos se enfocan en la comprensión para la apropiación del deporte como práctica socializadora, higienista y/o hedonista (plazas de deportes y clubes amateurs).

- Los intereses emancipatorios se enfocan en la transmisión, comprensión y problematización del deporte como fenómeno social y cultural (menester excluyente de la educación física en el contexto de la educación formal) (Corbo, Sarni, y Oroño, 2020).

\section{Conclusiones}

A raíz de lo que hemos venido expresando, entendemos que existe una dimensión ética que liga inexorablemente la producción de conocimiento con las posibilidades de transformación sobre la praxis a partir de la determinación de la factibilidad (Dusell, 2016), es decir, del lugar de la posibilidad en el hacer de la educación y por tanto de la educación física (y del deporte). Entendemos como Dusell que toda ética es crítica, a diferencia de lo moral que cae en el lugar de lo no pensado, de lo instalado como principio normativo.

Tomamos como punto de partida tres de los aspectos que venimos desarrollando para comenzar la discusión en cuanto a la enseñanza del deporte: 1) el saber de deporte es mucho más que el saber sus técnicas, tácticas y reglamentos; 2) su enseñanza debe pensarse desde la complementariedad necesaria en la producción de conocimiento en y sobre deporte y sobre enseñanza del deporte; y 3) las discusiones sobre la enseñanza del deporte deben atender a sus aspectos pedagógicos (sus sentidos educativos), al igual que a sus aspectos didácticos (las formas y sentidos de su enseñanza).

Por lo anterior... la pedagogía y la didáctica son indisociables: excluir la discusión de la enseñanza del deporte de la discusión sobre el cómo hacerlo, lo despoja de sus sentidos sociales y culturales y por tanto de sus relaciones con los fenómenos políticos y económicos que lo determinan, lo desconoce como objeto de praxis.

Pensar al deporte como objeto de enseñanza de la educación física implica superar la educación del practicante y pensar a su vez en la educación del consumidor y el espectador deportivo, el ciudadano que es parte de una sociedad que produce deporte y que es producida por él, en una educación deportiva (Velázquez Buendía, 2004). Es ahí entonces donde la acción de enseñanza demanda de la complementariedad de las ciencias que trabajan separadas - pero dialécticamente dependientes- en la producción de conocimiento en y sobre deporte.

En definitiva, entendemos que el deporte debe enseñarse en su dimensión concreta, comprendiendo las formas en que las relaciones estructurales que se tejen fuera 
de él - las relaciones de producción- condicionan lo que sucede en su interior tanto como configuran el deporte con el que convivimos.

La enseñanza del deporte debe pensar al docente como principal agente de transformación, enfocada en la habilitación de los estudiantes como potenciales agentes de cambio sobre la cultura deportiva.

La producción de teoría sobre el deporte, aunque construya de lo abstracto a lo concreto, es insuficiente cuando no vuelve sobre la praxis, cuando la relación dialéctica se interrumpe, cuando se cosifica al objeto.

Por último... dado que lo abstracto y lo concreto son indisociables, la teoría que no transforma, que no vuelve sobre la praxis, por más crítica que pretenda ser, terminará favoreciendo la reproducción.

Comprender el deporte para transformarlo puede aportar a la comprensión del mundo y sus relaciones y, por tanto, a su transformación para el mundo.

\section{Referencias bibliográficas}

Adorno, T. (1998). Educación para la emancipación. Madrid: Morata.

Adorno, T. (2015). Filosofía y sociología. Buenos Aires: Eterna Cadencia.

Adorno, T. (2017). Ontología y dialéctica: Lecciones sobre la filosofía de Heidegger. Buenos Aires: Eterna Cadencia.

Adorno, T., y Horkheimer, M. (2016). Dialéctica de la ilustración. Madrid: Akal.

Adorno, T., y Horkheimmer, M. (2014). Hacia un nuevo manifiesto. Buenos Aires: Eterna Cadencia.

Althusser, L. (2015). Iniciación a la filosofía para no filósofos. Buenos Aires: Paidós.

Aristóteles. (2019). Ética a Nicómaco. En Aristóteles, Ética a Nicómaco/Ética a Eudemo (pp. 128-406). Barcelona: Gredos.

Corbo, J. L., Sarni, M., y Oroño, M. (2020). Didáctica de la educación física escolar: Aproximaciones deontológicas. En E. Fiore y J. Leymonié, Didáctica práctica para enseñanza básica, media y superior (pp. 521-542). Montevideo: Grupo Magró.

Bracht, W. (2005). Cultura corporal, cultura de movimento ou cultura corporal de Movimento. En M. Souza Junior (org.), Educação física escolar: Teoria e política curricular, saberes escolares e proposta pedagógica (pp. 97-106). Recife: EDUPE.

Chevallard, Y. (1998). La transposición didáctica. Madrid: Aique.

Dusell, E. (2016). 14 tesis de ética. Madrid: Trotta.

Elias, N., y Dunning, E. (1992). Deporte y ocio en el proceso de la civilización. Madrid: Fondo de Cultura Económica.

Freire, P. (1969). La educación como práctica para la libertad. Buenos Aires: Siglo XXI. Gómez, J. (2002). La educación física en el patio. Buenos Aires: Stadium. 
Gramsci, A. (2015). Antología. México: Siglo XI.

Grundy, S. (1987). Producto o praxis curricular. Madrid: Morata.

Habermas, J. (1986). Conocimiento e interés. Madrid: Taurus.

Habermas, J. (1995). Teoría y praxis. Barcelona: Altaya.

Jameson, F. (2013). Valencias de la dialéctica. Buenos Aires: Eterna Cadencia.

Marx, C. (1989). Contribución a la crítica de la economía política. Moscú: Progreso.

Marx, C. (1969). Tesis sobre Feuerbach. En C. Marx y F. Engels, Obras escogidas (pp. 2629). Moscú: Progreso.

Marx, C., y Engels, F. (1978). Textos sobre educación y enseñanza. Madrid: Comunicación.

Sarni, M., y Corbo, J. L. (2021). El sentido del contenido deporte en el programa de Educación Primaria (Uruguay). Revista Universitaria de la Educación Física y el Deporte, 1(14). Recuperado de http://revistasiuacj.edu.uy/index.php/rev1/article/view/181.

Velázquez Buendía, R. (2004). Enseñanza deportiva escolar y educación. En A. Fraile Aranda (coord..), Didáctica de la educación física: Una perspectiva crítica y transversal (pp. 171-196). Madrid: Biblioteca Nueva. 